\title{
Preliminary Through-Out Research on Parallel-Based Remote Sensing Image Processing*
}

\author{
Guoqing $\mathrm{Li}^{1}$, Yan $\mathrm{Ma}^{2}$, Jian Wang ${ }^{1}$, and Dingsheng Liu ${ }^{1}$ \\ ${ }^{1}$ Key laboratory, Remote Sensing Satellite Ground Station, Chinese Academic of Sciences \\ No. 45 BeiSanHuanXi Road, P.O. Box 2434, Beijing, 100086, China \\ Graduate University, Chinese Academic of Sciences \\ No. 45 BeiSanHuanXi Road, P.O. Box 2434, Beijing, 100086, China \\ \{gqli, yma, jwang, dsliu\}@ne.rsgs.ac.cn
}

\begin{abstract}
As the most important and most complex Geo-Information, remote sensing data can be fast processed with cluster-based parallel computation technologies. The through-out ability of every step of such processing will affect heavily the application of remote sensing image parallel processing technologies. This paper shall discuss more detail how to set up the through-out model of remote sensing image parallel processing and presents some deeply research works on such through-out mechanism. A lot of experiments have been given to support a quantitative analysis method to adjust the performance of remote sensing parallel processing system.
\end{abstract}

\section{High Through-Out Computation and Remote Sensing Image Processing}

There are many research works focusing on how to improve the spatial data processing speed. HPC (high performance computation), especially based on cluster can bring the outstanding speed-up [1] [2]. On the other hand, HTC (high throughout computation) should also be paid more attention to [3].

The aim to research HTC is to build an economical and high efficiency system. High performance parallel computation can support the improvement on process speed of independent processing steps. However, the isolated effort on special step will not always cause the appearance of HTC. When we are building a HTC system, what we have to pay more attention to is the discovery and solver of bottlenecks.

Most important, remote sensing image processing has high data complexity. To design a parallel model, the matching relationship of data transfer, catching and node computing ability should be considered firstly. During certain time, HTC system can deal with most data than other system, and data flux is thought as the most important technical index for some applications.

The above research is supported by the National Key Research and Development Plan of China. 


\section{The Through-Out Rate Model of Remote Sensing Image Processing}

\subsection{Process Course Model}

A remote sensing image parallel process course is organized with some important steps, data loading, distribution, processing, collection and export, as shown in Fig. 1. Because the step of data loading is similar with data export, the step of data distribution is similar with data collection in the term of through-out character; such steps can be described with the steps of data loading and data distribution.

\section{Through-out rate model}

The through-out rate $\mathrm{R}$ is the most important index of HTC.

$$
T=T_{\text {input }}+T_{\text {dist ribute }}+T_{\text {process }}
$$

Where $T$ means the full process time, $T_{\text {input }}$ means the data loading time, $T_{\text {dist ribute }}$ means the data distributing time and $T_{\text {process }}$ means data processing time. If we define $\mathrm{M}$ is the amount of processed data, then

$$
\begin{gathered}
T_{\text {input }}=\frac{M}{R_{\text {ipput }}} \\
T_{\text {dist ribute }}=\operatorname{MAX}\left\{\frac{M_{i}}{R_{\text {distributi on }}}\right\} \quad i=1 \ldots n \\
T_{\text {process }}=\operatorname{MAX}\left\{\frac{M_{i}}{R_{\text {process }}}\right\} \quad i=1 \ldots n
\end{gathered}
$$

If we suppose data $\mathrm{M}$ is averagely distributed on $\mathrm{n}$ nodes, then $M_{i}=\frac{M}{n}$. From formula (1) to (4),

$$
T=\frac{2 M}{R_{\text {input }}}+\frac{2 M}{n R_{\text {distributi on }}}+\frac{M}{n R_{\text {process }}}=\frac{M}{n}\left(\frac{2 n}{R_{\text {input }}}+\frac{2}{R_{\text {distributi on }}}+\frac{1}{R_{\text {process }}}\right)
$$

Where $R_{\text {input }}$ is the data loading through-out rate, and $R_{\text {distribution }}$ means the data distribution through-out rate and $R_{\text {process }}$ is the rate of data processing.

$$
R=\frac{M}{T}=\frac{n}{\frac{2 n}{R_{\text {input }}}+\frac{2}{R_{\text {distributi on }}}+\frac{1}{R_{\text {process }}}}=\frac{1}{\frac{2}{R_{\text {input }}}+\frac{2}{n R_{\text {distribut on }}}+\frac{1}{n R_{\text {process }}}}
$$

If $R_{\min }=\operatorname{MIN}\left\{R_{\text {input }}, R_{\text {distribution }}, R_{\text {process }}\right\}, \quad R_{\max }=\operatorname{MAX}\left\{R_{\text {input }}, R_{\text {distribution }}, R_{\text {process }}\right\}$, there are the following conclusions: (1)When Rmin $\sim$ Rmax, the process course is in the condition of ideal through-out. In this condition, the improvement of system 
through-out is depended on the matching of these three through-out rates; however the limit of such improvement is not above one order of magnitude. (2)When Rmin $<<\operatorname{Rmax}$, the process course is in the condition of abnormal through-out. In this condition, the improvement of system through-out is depended on the tuning of the lowest course of through-out. (3)With the increasing of node number $n$, the throughout rate $\mathrm{R}$ is increasing. When $\mathrm{n}$ is large enough, $\mathrm{R}$ is limited in $R_{\text {input }}$ It means that the increasing of computing node will bring up the improvement of system throughout capability, while the limit of such increasing is $R_{\text {input }}$.(4)The through-out rate R is stable in some degree, which means $\mathrm{R}$ has low relativity with the scale of input data.
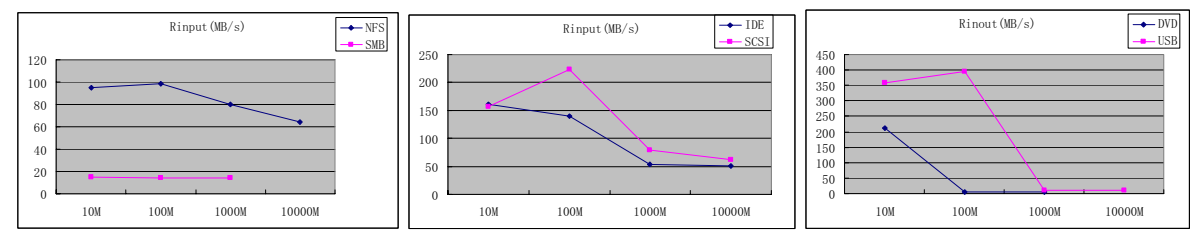

Fig. 2. The $R_{\text {input }}$ trend of three data loading methods

\subsection{Experiments}

Some experiments have been taken to research the performance and character of different stage. Fig. 2 shows three methods of data loading and exporting, which are local disk method (SCSI, SATA and IDE etc.), local extend storage method (DLT, DVD and USB etc.) and network method (NFS, SMB and RCP etc.). In Fig. 3, the different scale data in cache node is distributed to the other 8 nodes in Giga LAN with methods of MPI, NFS and PIPFS [4] Fig. 4 gives the comparison of the throughput between two typical remote sensing image algorithms FFT and Wavelet within same parallel environment.

\section{The Conclusion and Analyses of Through-Out Rate Model}

(1) When $\mathrm{nQ}$ is least than the range of $40 \sim 70 \mathrm{MB} / \mathrm{s}, \quad \mathrm{R}$ is limited by the throughput $\mathrm{nQ}$ and the capability of through-out can be simply accelerated with adding computing nodes.

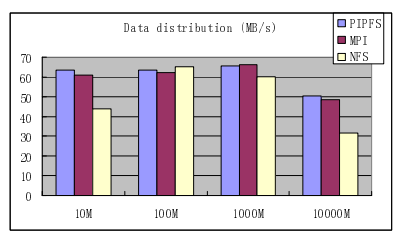

Fig. 3. the $R_{\text {distribution }}$ of three distribution mode

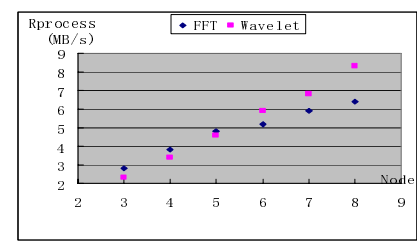

Fig. 4. $R_{\text {process }}$ trend of two typical processes

(2) When $\mathrm{nQ}$ is in the range of $40 \sim 70, \mathrm{R}$ is near $40 \sim 70 \mathrm{MB} / \mathrm{s}$. The better through-out performance can be implemented by adjusting the relationship of three courses. 
(3) When the nQ value is more than 70 , adding computing node number will not bring the increasing of R. In such system, it is no sense to extend the scale of computer cluster.

(4) When Q is large enough, which means the process algorithm is too simple, the most cost of the process courses is spend during data loading and distributing. Only little time cost is used in the data processing. The method to improve the through-out performance of such system is depended on the accelerating of data loading and distributing, which can be accessed with advance storage technologies and combined bandwidth technology.

(5) When $Q$ is not very large, the process algorithm is very complex and the process course will spend relative long time. The method to improve the through-out performance can be realized with the adding of computing nodes. The devoting of the improvement on storage technology will not

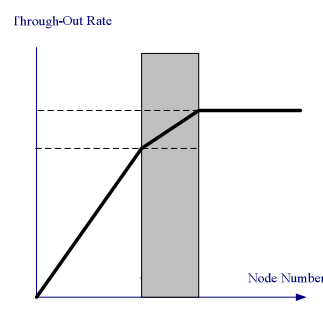

Fig. 5. The relationship between through-out rate and node number be outstanding in such condition.

In some degree, the above analyses are the predigesting of process courses. It will be more complex in real, for example the imbalance of load and data distribution. However, the methodology of through-out analyses is also useful for the research of such condition.

\section{References}

[1] High Performance Computing Clusters, Constellations, MPPs, and Future Directions, Jack Dongarra, Thomas Sterling, Computing in Science and Engineering, March/April 2005 (Vol. 7, No. 2) pp. 51-59

[2] Guoqing Li, Dingsheng Liu, Key Technologies Research on Building a Cluster-based Parallel Computing System for Remote Sensing, LNCS 3516, pp. 484-490

[3] IBM website:http://www-128.ibm.com/developerworks/cn/linux/cluster/hpc/part1/

[4] Keying Huang, Guoqing Li, Dingsheng Liu A parallel file system based on spatial information object, LNCS 3779, pp. 154-162 